\title{
eNeuro
}

Research Article: New Research / Disorders of the Nervous System

\section{Biophysical modelling of dopaminergic denervation landscapes in the striatum reveals new therapeutic strategy}

https://doi.org/10.1523/ENEURO.0458-21.2022

Cite as: eNeuro 2022; 10.1523/ENEURO.0458-21.2022

Received: 28 October 2021

Revised: 22 December 2021

Accepted: 10 January 2022

This Early Release article has been peer-reviewed and accepted, but has not been through the composition and copyediting processes. The final version may differ slightly in style or formatting and will contain links to any extended data.

Alerts: Sign up at www.eneuro.org/alerts to receive customized email alerts when the fully formatted version of this article is published.

Copyright @ 2022 Heltberg et al.

This is an open-access article distributed under the terms of the Creative Commons Attribution 4.0 International license, which permits unrestricted use, distribution and reproduction in any medium provided that the original work is properly attributed. 
1 Title:

2 Biophysical modelling of dopaminergic denervation landscapes in the striatum reveals new therapeutic strategy

3

4 Abbreviated title:

5 Modelling of dopaminergic denervation landscapes

23

\section{Authors and affiliations:} N. Rasmussen ${ }^{6}$

10

\section{Author contributions}

\section{Corresponding author email address:}

\section{Number of figures: 5}

\section{Number of words:}

Abstract: 165

Significance statement: 113

Introduction: 554

Discussion: 1,514
Mathias L. Heltberg1,2,3, Hussein N. Awada4, Alessandra Lucchetti², Mogens H. Jensen², Jakob K. Dreyer ${ }^{5}$ and Rune ${ }^{1}$ Laboratoire de Physique, École Normale Supérieure, 75231 Paris Cedex 05, France. ${ }^{2}$ Niels Bohr Institute, University of Copenhagen, 2100 Copenhagen, Denmark. ${ }^{3}$ Section of Surgical Pathophysiology, University Hospital Copenhagen, 2200 Copenhagen, Denmark. ${ }^{4}$ Department of Anesthesiology, University Hospital Copenhagen, 2200 Copenhagen, Denmark. ${ }^{5}$ Department of Bioinformatics, H Lundbeck A/S, 2500 Valby, Denmark. ${ }^{6}$ Center for Translational Neuromedicine, University of Copenhagen, 2200 Copenhagen, Denmark.

M.L.H., H.N.A., M.H.J, J.K.D., and R.N.R. designed the research; M.L.H., and A.L. performed the research; M.L.H., H.N.A., A.L., J.K.D., and R.N.R. analyzed the data; M.L.H., H.N.A., M.H.J., J.K.D., and R.N.R. wrote the paper.

Correspondence should be addressed to rune.nguyen.rasmussen@sund.ku.dk (R.N.R.) 


\section{Acknowledgements:}

32 We thank Ubadah Sabbagh, Akihiro Matsumoto, and Eric Nicholas for critical comments on the manuscript.

33 M.L.H. is grateful to Aleksandra Walczak and Thierry Mora for scientific discussions and valuable support. M.L.H.

34 thanks Angela Taddei and Judith Mine-Hattab for encouragement and a fantastic scientific environment. M.L.H.

35 and H.N.A. would like to thank Lene Oddershede for inspiration in the early stages of the project.

36

37 Conflict of interest statement:

38 The authors declare no conflict of interests.

\section{Funding sources:}

41 R.N.R. acknowledges support from the Lundbeck Foundation (R230-2016-2326); M.L.H. and M.H.J. acknowledge 42 support from the Danish Council for Independent Research and StemPhys DNRF Center of Excellence 43 (DNRF116), and M.L.H. acknowledges support from the Lundbeck Foundation (R347-2020-2250). 


\section{Abstract}

47 Parkinson's disease (PD) results from a loss of dopaminergic neurons. What triggers the break-down of 48 neuronal signaling, and how this might be compensated, is not understood. The age of onset, progression and 49 symptoms vary between patients, and our understanding of the clinical variability remains incomplete. In this study, we investigate this, by characterizing the dopaminergic landscape in healthy and denervated striatum, 51 using biophysical modelling. Based on currently proposed mechanisms, we model three distinct denervation patterns, and show how this affect the dopaminergic network. Depending on the denervation pattern, we show how local and global differences arise in the activity of striatal neurons. Finally, we use the mathematical 54 formalism to suggest a cellular strategy for maintaining normal dopamine signaling following neuronal denervation. This strategy is characterized by dual enhancement of both the release and uptake capacity of dopamine in the remaining neurons. Overall, our results derive a new conceptual framework for the impaired dopaminergic signaling related to PD and offers testable predictions for future research directions.

\section{Significance Statements}

60

Parkinson's disease, caused by a loss of dopaminergic neurons, is the second most common neurodegenerative disorder worldwide. Clinically, the age of onset, disease progression, and symptoms are highly variable between patients. Despite this, an understanding of the underlying mechanisms causing this variability is still missing. We here use biophysical modelling and show that the spatial pattern of dopaminergic denervation profoundly affects the anatomy and signaling of the dopaminergic network. We further show that the pattern of denervation has functional consequences for the activity of the downstream projection neurons, critical for the direct and indirect pathways. Our findings are useful in understanding the clinical variability of Parkinson's disease and offers several experientially testable predictions. 
Parkinson's disease (PD) is a common neurodegenerative disorder, affecting 1\% of people over the age of 60 worldwide (Hirtz et al., 2007). The disease is caused by a progressive loss of dopaminergic neurons in the substantia nigra pars compacta (SNc) (Damier et al., 1999; Rodriguez-Oroz et al., 2009), and symptoms typically emerge when $60-80 \%$ of these neurons are lost (Fearnley and Lees, 1991; Lee et al., 2000). Notably, the age of onset, disease progression, response to treatment, and symptoms are highly variable between patients (Greenland et al., 2018; Lewis et al., 2005), pointing to a complex relationship between neuron loss and PD etiology that remains to be understood.

Dopaminergic SNc neurons send projections to the dorsal striatum in the basal ganglia (Fig. 1A), an important area for motor function and executive control (Kreitzer and Malenka, 2008). These projections promote movement by modulating the excitability of GABAergic striatal spiny projection neurons (SPNs) by activating D1- or D2-class dopamine (DA) receptors (Kreitzer, 2009; Surmeier et al., 2007). DA increases the excitability of D1 receptor-expressing SPNs (D1-SPNs) and decreases the excitability of D2 receptor-expressing SPNs (D2SPNs) (Kreitzer and Malenka, 2008; Lahiri and Bevan, 2020). D1- and D2-SPNs are critical components of two distinct pathways, traditionally thought to control movements in opposing ways: the direct pathway promotes desired movements while the indirect pathway suppresses unwanted movements (Cui et al., 2013; Freeze et al., 2013; Kravitz et al., 2010; Kreitzer and Malenka, 2008) (Fig. 1B). In PD, dopaminergic neurons are progressively lost, leading to striatal DA depletion, abnormal SPN activity and movement deficits (Kravitz et al., 2010; Mazzoni et al., 2007; Rodriguez-Oroz et al., 2009). Despite the central role of failing DA signaling in PD etiology, little is known about the nature of striatal DA signaling before and during disease progression, posing a significant obstacle to the development of therapeutic strategies which maintain normal DA signaling in PD patients. have proposed different mechanisms, including the prion-hypothesis (Chu and Kordower, 2015; Prusiner, 2012) and oxidative stress (Jenner, 2003; Sulzer, 2007). However, little attention has been given to investigating the spatial and temporal patterns of dopaminergic neuron loss. Clinical imaging techniques, measuring DA transporter densities, provide a correlate of dopaminergic innervation (Ba and Martin, 2015; Wang et al., 2012), 
but cannot resolve the fine-scale organization of neurons at cellular resolution. In animal models, neuronal firing and DA signals can be recorded invasively (Lippert et al., 2019; Patriarchi et al., 2018) and correlated with dopaminergic neuron density postmortem. In addition to the challenge of being limited to a highly localized area, this approach lacks the temporal scale needed to track slow changes in neuron density and DA signaling.

Here, we developed a series of biophysical models to study how signals are lost by the denervation of dopaminergic neurons. Our results support a conceptual framework where the clinical manifestations of PD are rooted in the distinct denervation patterns and, importantly, provide theoretical predictions to be experimentally tested. Specifically, our work predicts that 1) variability in PD progression and symptoms stems from different spatiotemporal striatal denervation patterns caused by distinct cellular disease mechanisms, and 2) a dual cellular strategy, enhancing both release and uptake capacity of dopamine in remaining neurons, can counteract striatal signaling disruption caused by dopaminergic denervation.

\section{Materials and Methods}

A detailed description of all mathematical derivations and formulations, biophysical models, and algorithms are included in the Extended Data Equations. In brief, this work uses numerical and analytical mathematical methods to theoretically investigate and characterize the dopaminergic innervation of the human striatum, and the networks that break down following denervation. In the first part of the paper, we use mean-field theory to derive a differential equation describing dopamine (DA) signaling in a mesoscopic region of the striatum. By inducing three distinct periods of dopaminergic neuron firing, we solve this numerically. Following this, we introduce individual axonal arbors in the striatum, using the random generator applied in MATLAB. To characterize the organization and spatial coverage of arbors we analyze whether innervation was coherent within the striatum. Inspired by the mathematical analysis of communication classes, we quantify the number of what we termed contiguous arbor classes (CACs) and estimate the unoccupied space by placing 10,000 random points in the striatum. Next, we repeat these measures for the three denervation mechanisms: random denervation (RD), prion-like denervation (PLD), and stress-induced denervation (SID) (described in further detail below). We then introduce a differential equation describing cyclic adenosine monophosphate (cAMP) following DA stimulation, which we solve numerically. With this, we record the maximal cAMP value for both 
D1- and D2-SPNs. We next implement a Hodgkin-Huxley-inspired neuronal model and simulated this in Python using the Numba package. Using this, we measure the number of elicited action potentials in a short temporal window, in which the D1- and D2-SPNs were stimulated with the corresponding maximal cAMP level. This was done for 100 neurons at several different levels of denervation. Finally, we use the average number of action potentials recorded in this window as input for 10,000 randomly positioned points in the striatum, dependent on the denervation model and levels. Using these numbers, we calculate a spatial average and standard deviation of the maximal firing rates of D1- and D2-SPNs. In this work, we did not employ statistical significance testing to compare conditions since all results were derived from analytical simulations and thus the major source of uncertainty is inherent to the chosen parameter values rather than variance across simulation iterations. All code is made publicly available on a GitHub repository: https://github.com/Mathiasheltberg/Theoretical_Denervation_ParkinsonsModel.

\section{Results}

\section{Functional and spatial characterization of DA signaling in the healthy striatum}

We began our investigation by modelling DA signaling in the fully innervated human striatum, specifically the putamen, which we defined as the healthy state (Dreyer, 2014; Dreyer et al., 2010). We simulated the firing of dopaminergic SNc neurons and described the extracellular DA concentration. For this, we employed a model describing DA in a subvolume of $10^{3} \mathrm{~mm}^{3}$ (see Extended Data Equations). Given the estimated density of $\sim 0.1$ dopaminergic axonal terminals per $\mathrm{mm}^{3}$ in the healthy striatum (Doucet et al., 1986; Dreyer, 2014; Dreyer et al., 2010), this volume contains on average 100 terminals, each of which was treated as an individual element. This is a reasonable approximation since each time a neuron fires, only a fraction of its terminals release transmitter. Estimates of the vesicular release probability of dopaminergic terminals are thus within the range of 6-20\% (Dreyer, 2014; Pereira et al., 2016), and only 30\% of the terminals may contain the molecular machinery for exocytosis (Liu et al., 2018). Based on this, we employed a deterministic mean-field model that approximates DA inside the $i^{\prime}$ th subvolume as:

$$
\frac{d M_{i}}{d t}=\Delta v N_{i}-V_{M} N_{i} \frac{M_{i}}{K_{M}+M_{i}}+\left(D \nabla^{2} M_{i}-\epsilon M_{i}\right)
$$


150

151

152

153

154

155

156

157

158

159

160

161

162

163

164

165

166

167

168

169

170

171

172

173

174

175

176
Here $M$ is the DA concentration, $\Delta$ is the amount of DA released by a terminal, $v$ is the neuronal firing frequency, $V_{M}$ is the DA uptake per terminal, and $N$ is the number of terminals within the subvolume. DA remains active in the extracellular space until it is removed by either transporters or degraded enzymatically (Fig. 1A), so we modeled transporter-mediated DA uptake after the Michaelis-Menten uptake equation. We also included a simple degradation term $(\epsilon)$, and a term to account for the diffusion between neighboring subvolumes $(D)$. These are, however, so small that they can be neglected if a region has dopaminergic innervation and will only be considered for regions deprived of terminals, and hence they are placed in parentheses. As shown in previous studies, we included that each dopaminergic neuron can express one of three firing patterns: pauses, tonic or phasic (Grace and Bunney, 1984b, 1984a). Thus, in the model, the firing frequency could take one of three values: 0 (pauses), $4 \mathrm{~Hz}$ (for tonic) or $20 \mathrm{~Hz}$ (for phasic). All employed parameter values (Extended Data Fig. 11) were adopted from previous theoretical work which used experimentally determined measurements to constrain their model (Dreyer et al., 2010, see Table 1). From this, we obtained DA time courses that clearly reflected the underlying neuronal firing patterns, exhibiting periods of tonic and phasic DA signaling, and pauses where DA is cleared from the extracellular space (Fig. 1C).

We next characterized the dopaminergic innervation of the striatum at a mesoscale. To mimic the shape of the putamen in the human striatum, we modeled it as an ellipsoid. Dopaminergic innervation was constructed by filling the volume with axonal arbors from $10^{5}$ SNc neurons, based on estimates from human SNc (Hardman et al., 2002) and the fact that these neurons have wide-spread projection targets (Poulin et al., 2018). Each neuron contributed with a spherical arbor with a radius of $0.5 \mathrm{~mm}$ (which is our best estimate based on the existing data), wherein the density of terminals was constant (Doucet et al., 1986; Matsuda et al., 2009). To characterize the organization and spatial coverage of arbors within the striatum, we analyzed whether innervation was coherent within the striatum. This can be quantified by the number of what we termed contiguous arbor classes (CACs) - inspired by the mathematical analysis of communication classes. We assumed that dopaminergic neurons belonged to the same CAC if their arbors considerably overlapped (their arbor centers less than $0.5 \mathrm{~mm}$ apart; Fig. 1D). Hence, if the number of CACs is low, it suggests a high degree of spatial coverage and cohesion, and vice versa. For classifying neurons into CACs, we used a Markov chain-inspired algorithm (see Extended Data Equations). We found that all neurons in the healthy striatum belonged to the same CAC (Fig. 1E), 
177

178

179

180

181

182

183

184

185

186

187

188

189

190

191

192

193

194

195

196

197

198

199

200

201

202

suggesting a high degree of coverage (Fig. 1F). For each neuron, we also counted the number of overlapping arbors, and this metric followed a Poisson distribution (Fig. 1G).

From the equation above, it is evident that decreasing $N_{i}$ does not affect the steady state DA concentration considerably unless this value is approximately zero. From calculations on the diffusion equation (see Extended Data Equations), we determined that each point within the striatum with a distance larger than $0.1 \mathrm{~mm}$ to its nearest neighboring arbor was defined as isolated. We therefore searched for spatially isolated areas where the innervation was sparse, since such areas would be more susceptible to impairments in DA signaling during denervation. Using Monte Carlo simulations, we approximated the distribution of smallest distances and used Voronoi tessellation to find the most isolated points (Fig. 1H; see Extended Data Equations). This showed that no isolated areas existed in the fully innervated striatum of our model. This result is dependent on the size of the arbors as well as their numbers in the healthy striatum, and we thus tested different values of both and found the system to be quite robust.

These results demonstrate that the modelled dopaminergic arbors comprise a network that densely covers the striatum, where no isolated areas exist.

\section{Different denervation patterns break down the dopaminergic network with distinct evolutions}

In biology, structure often informs function. We therefore probed the spatial landscape of dopaminergic arbors in the denervating model striatum. The molecular pathways involved in the loss of dopaminergic neurons are beyond the scope of this study. Instead, we sought to characterize the organization of the remaining innervation arising from distinct models of progressive neuron loss.

To describe denervation, we assumed that all neurons have a rate of dying. All models were simulated using the Gillespie algorithm, and neurons were removed according to their rate. We first modelled the denervation to be independent of the spatial position of a neuron, meaning that all neurons had the same rate of dying. This we termed random denervation (RD; Fig. 2A). The second model, prion-like denervation (PLD; Fig. 2B), one neuron was initially infected. All infected neurons then had a rate to infect others and dying, while all uninfected neurons had zero rate of dying. Based on the rates, one infected neuron was chosen to infect two neighboring 
203

204

205

206

207

208

209

210

211

212

213

214

215

216

217

218

219

220

221

222

223

224

225

226

227

228

229 neurons before being removed from the network. This algorithm was based on the proposed mechanisms where protein aggregates spread between neurons and cause their degeneration (Chu and Kordower, 2015; Prusiner, 2012; Surmeier et al., 2017). In the third model, stress-induced denervation (SID), each neuron has a rate of dying calculated as a sigmoidal function of its number of neighbors. Thus, neurons with few overlapping arbors have a higher risk of dying compared to neurons with many overlapping arbors. This algorithm was based on the proposed mechanism where the remaining neurons may upregulate their DA synthesis and firing activity to maintain DA signaling. However, these neurons may already be close to their maximal metabolic capacity (Bolam and Pissadaki, 2012), and increased activity could trigger stress-induced degeneration (Jenner, 2003; Sulzer, 2007). We want to emphasize, that we do not consider these three models as mutually exclusive nor the "ground truth" mechanisms for the process of dopaminergic denervation. However, they each represent a simple algorithm for studying the self-organization of these complex phenomena and has the potential to give important insight in the different denervation structures.

Using simulations, we observed how the three models resulted in distinct spatial landscapes, each characterized by a unique dopaminergic network breakdown (Figs. 2D-2G). For RD, the remaining arbors covered the entire striatal space but no longer belonged to the same CAC. In contrast, for PLD, large fractions of the striatum were deprived of arbors and instead dominated by one or two subregions with seemingly normal innervation. For SID, arbors were concentrated in small, isolated subregions, each forming its own CAC. We quantified these observations by the distribution of the number of overlapping arbors (Fig. 2E). For PLD, a notable fraction of arbors had very low numbers of overlapping arbors, whilst a larger fraction had numbers like those in the healthy striatum. In SID, only arbors with many overlapping neighbors remained. Importantly, a commonality of all models was that the dopaminergic network broke down into multiple CACs, but in distinct patterns (Fig. 2F): RD had only small classes remaining, PLD contained many small but also one dominating class, whereas SID contained many classes containing 100 or more arbors. We also assessed the emergence of isolated areas (Fig. 2G). For RD, no isolated areas existed. In contrast, for both PLD and SID, the striatum contained numerous isolated areas, deprived of arbors.

Next, we followed spatial characteristics as a function of denervation. First, we determined the percentage of remaining arbors as a function of time (Fig. $2 \mathrm{H}$ ). For RD, this followed an exponential decay with a relatively 
230

231

232

233

234

235

236

237

238

239

240

241

242

243

244

245

246

247

248

249

250

251

252

253

254

255

slow temporal progression. Interestingly, for PLD, the curve followed a convex function, suggesting that neuron loss accelerated with time, whereas the curve for SID followed a concave function, indicating that denervation in this scheme started fast, but then slowed with time. These results have predictive strength and can be mathematically described by stretched exponentials of the form: $N(t) \propto e^{-b t^{c}}$, with $b$ being the decay rate and $c$ $=1$ for RD, $c>1$ for PLD and $c<1$ for SID. We next characterized the breakdown of the spatial network, by calculating the fraction of arbors in the largest CAC (Fig. 2I). PLD kept one dominating class until the final stage of denervation, while RD and SID were characterized by a tipping point, at which the network dramatically transitioned from fully coherent to segregated into multiple classes. Notably, this transition occurred around 75\% denervation, which often correlates with the onset of symptoms in PD patients (Bernheimer et al., 1973; Fearnley and Lees, 1991; Lee et al., 2000; Ma et al., 1997). Finally, we probed the emergence of isolated areas, practically devoid of DA signaling. At $75 \%$ denervation, isolated areas comprised $\sim 50 \%$ and $20 \%$ of the striatum in the PLD and SID models, respectively (Fig. 2J). At the same denervation level, no isolated areas existed for RD, but these emerged at severe denervation.

Overall, we found notable spatial and temporal differences between distinct models of dopaminergic denervation. These differences between models were remarkably robust to changes in key parameters, that is, axonal arbor volume and numbers of dopaminergic neurons in the healthy state (Extended Data Fig. 2-1).

\section{Dopaminergic denervation affects cAMP production and the activity of striatal SPNs}

The excitability of SPNs is, along with several other factors, strongly regulated by DA (Kreitzer, 2009; Kreitzer and Malenka, 2008; Lahiri and Bevan, 2020; Surmeier et al., 2007). Thus, we next asked how dopaminergic denervation affects the activity of individual SPNs. Previous work has shown that D1 and D2 receptors have low and high DA affinity, respectively (Richfield et al., 1989) (Fig. 3A). DA regulation of SPN excitability is mediated by the signaling molecule cyclic adenosine monophosphate (cAMP). D1 and D2 receptor activation increases and decreases the production of cAMP, respectively, and cAMP in turn regulates SPN ion channels (Kreitzer, 2009; Kreitzer and Malenka, 2008; Surmeier et al., 2007). Inspired by previous work (Dreyer et al., 2010), we described the intracellular cAMP concentration in D1- and D2-SPNs as: 
258 Here $\alpha$ is the steady state production of cAMP, and $\delta$ is its spontaneous decay. In addition, receptor-dependent 259 cAMP production was implemented: cAMP in D1- and D2-SPNs increased and decreased with DA stimulation, 260 respectively. With increasing denervation, cAMP production during phasic firing became progressively lower in 261 D1-SPNs, whilst in D2-SPNs it became progressively lower during firing pauses (Figs. 3B and 3C).

262 We next asked how these impairments in cAMP signaling may manifest in the activity of SPNs. For this, we used 263 a previously published Hodgkin-Huxley-inspired model (Rasmussen et al., 2017; Tatsuki et al., 2016) to simulate 264 the membrane potential $\left(\mathrm{V}_{\mathrm{m}}\right)$ of D1- and D2-SPNs. This model contains extrinsic and intrinsic ion channel 265 conductances. The extrinsic conductances are NMDA, AMPA, and $\mathrm{GABA}_{\mathrm{A}}$ ion channels. The intrinsic 266 conductances are voltage-gated and persistent $\mathrm{Na}^{+}$channels, voltage-gated $\mathrm{Ca}^{2+}$ channels (Cav), and voltage267 gated, leak, fast A-type, inwardly rectifying, slowly inactivating $\left(\mathrm{K}_{\mathrm{SI}}\right)$, and $\mathrm{Ca}^{2+}$-dependent $\left(\mathrm{K}_{\mathrm{Ca}}\right) \mathrm{K}^{+}$channels 268 (Rasmussen et al., 2017; Tatsuki et al., 2016) (see Extended Data Equations). Using this model, we could closely mimic the $V_{m}$ dynamics of SPNs. These neurons are characterized by their transitions between downstates and upstates (Sippy et al., 2015; Wickens and Wilson, 1998). In our model, upon increased levels of synaptic barrages (implemented by increasing the stochastic noise of the $V_{m}$ ), both D1- and D2-SPNs transitioned into a brief upstate in which multiple action potentials were fired (Figs. 3D and 3H).

273 To implement the effect of DA, via its regulation of cAMP, on the $V_{m}$ dynamics of D1- and D2-SPNs, we targeted 274 the high-threshold $\mathrm{Ca}_{\mathrm{v}}\left(\mathrm{N}\right.$ - and P-type), $\mathrm{K}_{\mathrm{S}}$, and $\mathrm{K}_{\mathrm{Ca}}$ channels, which are negatively influenced by DA and cAMP signaling (Lahiri and Bevan, 2020; Nisenbaum et al., 1994; Shen et al., 2004; Surmeier and Kitai, 1993; Surmeier et al., 1995, 2007). Thus, for increasing cAMP levels, the conductance of these channels decreases and vice versa. We note that other channels may also be subject to dopaminergic modulation, such as persistent $\mathrm{Na}^{+}$or $\mathrm{NMDA}^{-}$ channels, but to limit the parameter space, we here focus on the above mentioned $\mathrm{Ca}^{2+}$ and $\mathrm{K}^{+}$channels. For stimulating D1- and D2-SPNs, we used the cAMP concentrations observed during dopaminergic phasic firing and firing pauses, respectively. This was motivated by the result that, in the healthy striatum, the maximal cAMP production in D1- and D2-SPNs was observed during these two phases respectively (Fig. 3B). In itself, cAMP 
282

283

284

285

286

287

288

289

290

291

292

293

294

295

296

297

298

299

300

301

302

303

304

305

306

307

308

stimulation was very rarely sufficient to evoke a transition from the downstate to an upstate in types of SPNs (Figs. 3E and 3I), supporting the notion of DA as a "modulator" rather than a "driver" (Kreitzer, 2009; Lahiri and Bevan, 2020; Surmeier et al., 2007). However, if DA and cAMP stimulation coincided with increased levels of synaptic barrages, this triggered a robust upstate that lasted longer and elicited more action potentials than with synaptic barrages alone (Figs. 3F and 3J); this demonstrates that cAMP powerfully regulates the excitability of D1- and D2-SPNs. As a result, in the denervated state, the activity of SPNs was notably affected (Figs. 3G and $3 \mathrm{~K})$ : the duration of the upstate and the firing rate during the upstate were strongly diminished in both D1- and D2-SPNs. These findings were replicated using the simpler Izhikevich model (Extended Data Fig. 3-1), suggesting model-invariance.

Together, these data demonstrate that DA signaling, via its downstream effector cAMP, can regulate the firing activity of SPNs, and this regulation is impaired in the denervated state.

\section{Distinct denervation patterns differentially affect global SPN firing activity}

Next, we sought to investigate how different denervation patterns affect the activity of D1- and D2-SPNs across striatal space. For this, we spatially mapped the maximal firing activity for both types of neurons; D1-SPN during phasic firing and D2-SPN during firing pauses using the Hodgkin-Huxley-inspired model (Figs. 4A and 4B). In $\mathrm{RD}$, although almost all the subregions had relatively low DA levels compared with the healthy striatum, this was still sufficient to evoke intermediate D1-SPN firing rates across the extent of the striatum. In contrast, in both PLD and SID, D1-SPN firing was high only in the subregions with preserved DA innervation. Noticeably, PLD transformed the striatum into a strongly polarized activity map, whereas SID caused local heterogeneity. For D2-SPNs, the emergence of isolated areas, resulted in a very different outcome. Since the maximal DA concentration in isolated areas is zero (except for small diffusive fluctuations), D2-SPN firing rates were here very high, most profoundly expressed for PLD and SID. We note here that, under physiological conditions, D2SPNs residing in regions deprived of DA signaling might adapt by downregulating their firing rates to maintain homeostasis. In this scenario, the results would likely be like those for D1-SPNs.

Finally, we characterized SPN activity in the three denervation models as a function of denervation. The mean D1-SPN firing rates decreased linearly as a function of denervation in all models (Fig. 4C). We also noted that the 
321

322

323

324

325

326

327

328

329

compensation (ERC; Fig. 5A):

$$
\Delta_{+} \mapsto \frac{\Delta_{0}}{1-\delta}
$$

330 Here we introduced the compensation parameter $\delta$, a sigmoidal function going from zero to one as a function of 331 the number of dopaminergic arbors covering a small volume. The parameter $\Delta_{0}$ refers to the DA release in healthy subregions, whereas $\Delta_{+}$is the compensated release value. Second, DA transporters, expressed on 
333 terminals, may reduce their uptake capacity (Greenbaum et al., 2013; Lee et al., 2000; Zigmond, 1997; Zigmond et al., 1990). We refer to this as decreased uptake compensation (DUC; Fig. 5A):

$$
V_{-} \mapsto V_{0}(1-\delta)
$$

335 As above, the parameter $V_{0}$ refers to the uptake value in healthy subregions, whereas $V_{-}$is the compensated 336 uptake strength. Finally, we suggest a mechanism where neurons compensate by enhancing both DA release and uptake capacity in the terminals. This idea stems from the equations for DA concentration dynamics, from where we can show mathematically that this combination recovers the original equations. Such a compensatory mechanism has not previously been suggested, and we refer to this as dual enhanced compensation (DEC; Fig. $3405 \mathrm{~A}$ ); this is included in the model through changes in both the uptake and release parameters:

$$
\Delta_{+} \mapsto \frac{\Delta_{0}}{1-\delta} \text { and } V_{+}=\frac{V_{0}}{1-\delta}
$$

Here all parameters are defined as above.

342 With these implementations, we simulated DA signaling and the corresponding cAMP production in D1- and D2343 SPNs with $80 \%$ denervation. As shown above, in the absence of compensation, DA release during tonic firing is unaffected, but notably affected during phasic firing and firing pauses (Fig. 3B). Here, during tonic firing, the DA concentration notably increased for ERC and DUC models; during phasic firing, DA was increased for ERC, and during firing pauses, DA removal was incomplete for DUC (Fig. 5B). Importantly, the DEC model preserved DA levels during both tonic and phasic firing at comparable levels to in the healthy state, while still allowing complete DA removal during firing pauses (Fig. 5B). We also tested several postsynaptic compensatory mechanisms previously proposed in the literature, including increased D2 receptor expression (Guttman and Seeman, 1985), enhanced D1 and D2 receptor sensitivity (Lee et al., 1978), and suppressed cAMP degradation in D1 and D2-SPNs (Niccolini et al., 2015). These mechanisms were inadequate to restore DA and cAMP signals in SPNs (Extended Data Fig. 5-1), and we therefore did not explore these further.

354 activity in the denervated state (Figs. 3 and 4). For this we calculated the spatial mean and standard deviation of 355 the maximal D1- and D2-SPN firing rates in the three denervation models and combined these with the 
356

357

358

359

360

361

362

363

364

365

366

367

368

369

370

371

372

373

374

375

376

377

378

379

380

381

382

presynaptic compensation mechanisms (Fig. 5C). Interestingly, in the scenario of RD, only the DEC model preserved the mean level of D1- and D2-SPN firing rates. In contrast, for PLD and SID, none of the three compensation models were able to counteract the decrease in D1-SPN firing with denervation, whilst all models performed relatively well for D2-SPN firing activity. For the standard deviation of the D1-SPN firing rates, we note that in the RD scenario, all compensation models, as well as the non-compensation state, maintained this measure near the healthy level (Fig. 5D). In contrast, for PLD and SID, the standard deviation was notably increased for all compensation models, and curiously, the non-compensated state was most similar to the healthy state. For the standard deviation of the D2-SPN firing activity, none of the compensation models truly maintained this measure close to the healthy level, regardless of the denervation pattern (Fig. 5D). It is here worth noting that the DEC model, across all denervation patterns, maintained the standard deviation of D2-SPN firing activity at a very low level. This is because, in regions with low dopaminergic coverage, DA signaling from remaining terminals in the DEC model can compensate, restoring coherent neuronal activity. The low standard deviation in firing activity across neurons means that all striatal subregions are capable of generating a very similar firing response upon dopaminergic stimulation. Overall, we conclude that the DEC model, in combination with the RD pattern, best preserved the SPN firing activity. In the final set of simulations, we thus explored this for all levels of denervation (Fig. 5E). For the DEC model, the firing rates of both D1- and D2-SPNs remained remarkably close to the healthy state, despite reaching severe denervation. In contrast, for the ERC and DUC models, even at intermediate denervation, SPN firing differed from the healthy state. Therefore, the ERC and DUC mechanisms do not seem ideal as therapeutic strategies. These findings were replicated with the Izhikevich model (Extended Data Fig. 5-2).

Taken together, these results show that an ideal strategy to maintain normal SPN activity is to locally introduce a dual compensation mechanism — increasing both DA release and uptake capacity — and to globally minimize the dopaminergic arbor density differences, or at least avoid the emergence of isolated areas.

\section{Discussion}

In this work, we used biophysical and mathematical modeling to investigate the spatial and functional landscape of dopaminergic signaling in the healthy and parkinsonian striatum. First, we showed that the spatial pattern of 
383

dopaminergic denervation profoundly affects the structural and temporal breakdown of the dopaminergic network in the striatum. Second, we derived how the local and global activity of D1- and D2-SPNs were differentially affected as a function of the spatial dopaminergic denervation pattern. Third, we identified that a combination of enhanced DA release and uptake capacity, presents a feasible strategy for maintaining the normal striatal DA signaling, when neurons are progressively lost.

\section{Clinical variability may be mediated by different dopaminergic denervation patterns}

PD symptoms often present when $60-80 \%$ of dopaminergic neurons are lost (Fearnley and Lees, 1991; Ma et al., 1997). Still, the age of onset, disease progression and symptoms can vary notably between patients (Greenland et al., 2018; Lewis et al., 2005). We believe that the spatial pattern of denervation might play a role in this clinical variability. Our model shows that the dopaminergic denervation pattern may lead to different DA signaling landscapes. After an initial slow denervation rate, the loss of neurons accelerated with time in the PLD model. In contrast, in the SID model, denervation slowed with time after an initial rapid loss of neurons. The breakdown of CACs in the PLD and SID models correlates well with the clinical progression pattern seen in the early and middle stages of PD (Eckert et al., 2007; Liu et al., 2015). In patients with RD, disease progression may be slow, whereas in patients with PLD, the progression may accelerate rapidly. Hence, if the total density of striatal dopaminergic terminals could be measured as a function of time in the early stages of the disease, our model would predict the denervation pattern causing PD in individual patients. Assuming that different denervation landscapes stem from distinct molecular mechanisms within the dopaminergic neurons, a novel experimental technique called mass synaptometry (Gajera et al., 2019, 2021) could be exploited to obtain information about the molecular profile of the synapses in the remaining neurons; allowing the possibility to cross correlate the two predictions and ideally validating or rejecting our model. A possible limitation of this approach is that mass synaptometry was performed in vitro from postmortem brain samples (Gajera et al., 2019). Thus, if samples are obtained from PD patients after their death only, this would not necessarily reveal the molecular mechanisms in the early phases of the disease, so ideally, the optimal approach would be to obtain samples from patients while alive and as early as possible. This could potentially be obtained during neurosurgery for implanting deep brain stimulation electrodes. Furthermore, it may be feasible to, at least in 
part, predict the disease progression time course, and from that determine the ideal therapeutic strategy for the individual patient. We therefore propose that future clinical experiments aim to measure the density of dopaminergic terminals in the striatum of PD patients over time and their molecular characterization, using for example single-photon emission computed tomography (Ba and Martin, 2015; Wang et al., 2012) and mass synaptometry, and to correlate this to disease progression. Combining the results from such experiments with biophysical modelling would elucidate the molecular and network mechanisms causing PD and disease variability. We also found that the absolute time course of dopaminergic denervation was remarkably distinct between the different denervation patterns (Fig. 2), and this observation could potentially aid clinicians in determining the differential diagnosis of parkinsonism. Clinical imaging of early-stage PD patients has shown that structural innervation differences in the striatum, albeit embracing a notably larger striatal area than our results, relates to different PD-related diseases. For example, large-scale asymmetry in striatal dopaminergic innervation associates with idiopathic parkinsonism (Kim et al., 2002; Ziebell et al., 2012), while large-scale symmetric denervation associates with atypical parkinsonian syndromes such as supranuclear palsy (Filippi et al., 2006; Knudsen et al., 2004; Varrone et al., 2001; Ziebell et al., 2012). This difference might be, at least in part, explained by the three denervation patterns described in our work. Thus, the denervation curves from our model, in combination with high-resolution imaging and the mass synaptometry technique, might portent a valuable tool for distinguishing between different forms of parkinsonism in individual patients.

\section{Distinct denervation patterns may differentially affect the direct and indirect pathway}

When dopaminergic neurons were lost, the burst firing during upstates of D1- and D2-SPNs was severely impaired (Fig. 3). Given that D1- and D2-SPNs are critical components of the direct and the indirect pathways, respectively, it is plausible that these two pathways would be affected as a result. The reduction of firing in D1SPNs during phasic dopaminergic firing may complicate the initiation of voluntary movements, whilst the impaired firing in D2-SPNs during dopaminergic firing pauses may facilitate unwanted, involuntary movements. Given that, in the denervated striatum, DA signaling and SPN activity varied across space depending on the denervation pattern, we expect that different subregions of the striatum will have normal and abnormal activity of the direct and indirect pathways, depending on the denervation pattern. This may contribute to why disease 
symptoms can vary notably between PD patients. We mention this with the caveat that our simulations of SPN

438

439

440

441

442

443

444

445

446

447

448

449

450

451

452

453

454

455

456

457

458

459

460

461

462

463 activity have their limitations. For example, DA is not the only modulator of SPN activity; also, local GABAergic and cholinergic interneurons regulate the activity of SPNs (Burke et al., 2017; Kreitzer, 2009), and our simulations do not account for that. Furthermore, we here investigated the acute effects of dopaminergic denervation and our results do thus not take into account the long-term changes in glutamatergic synaptic activity that may develop in the striatum as a result of denervation (Kreitzer and Malenka, 2008; Surmeier et al., 2007), nor did we explore possible changes in dopaminergic autoregulation (Ford, 2014). Future work should aim to investigate the interplay between different dopaminergic denervation patterns and these other mechanisms regulating SPN activity, for example in a more comprehensive basal ganglia network model, as recently developed (Hjorth et al., 2020).

\section{A dual cellular strategy could promote normal DA signaling}

The most common pharmacological treatment for PD is to administer levodopa, with the goal of increasing DA levels within the brain (Hauser, 2009; LeWitt, 2008; Salat and Tolosa, 2013). However, not all patients respond well to this treatment, and some experience side effects with long-term treatment (LeWitt, 2008; Salat and Tolosa, 2013). During the early stages of PD, the dopaminergic neuron loss is believed to be counterbalanced by endogenous compensatory mechanisms (Brotchie and Fitzer-Attas, 2009; Zigmond, 1997). Knowledge of such mechanisms could reveal potential targets for novel therapeutic strategies (Brotchie and Fitzer-Attas, 2009). In our work, we found that DA signaling cannot be fully characterized merely by its tonic level, since the correct occurrence of peaks during phasic firing and the complete removal of DA during firing pauses likely plays important roles in proper neuronal signaling. Therefore, when evaluating the therapeutic potential of a cellular target, it is important to assess its effects on the full DA signaling spectrum. Through our investigation we found that the optimal approach to minimize the dopaminergic signaling effects of denervation, is to upregulate the released DA in combination with enhancing its uptake. While the first is typically achieved in patients by administering levodopa (Hauser, 2009), the latter has not, to our knowledge, been attempted yet, and could in principle be achieved by enhancing the level of dopamine transporters in the neurons. Therefore, this theoretical work suggests a new therapeutical strategy, where levodopa is given in combination with 
enhancement of dopamine transporters in order to maintain the steady state level of DA while at the same time recovering the fast changes in DA concentration during the short transient intervals of bursts and pauses. In contrast to other mechanisms, this dual mechanism preserved the DA signaling spectrum, without increasing tonic DA levels. We note that clinically this would involve a combinatorial treatment with, for example, levodopa and a genetic approach for boosting the synthesis of DA transports - which we believe will be possible to test in the future. Thus, if some neurons remain, this mechanism can restore the DA signaling properties, emphasizing the great importance of avoiding areas completely devoid of DA terminals. We hypothesize that this dual strategy might postpone the onset of severe symptoms by upholding normal DA signaling and could potentially cause less side effects since baseline DA is maintained at a comparable level to that in the healthy striatum.

\section{Conclusion}

In conclusion, our work constitutes a new conceptual model for the impairments of dopaminergic signaling related to PD. In this, we developed a holistic framework linking the activity of individual neurons to the spatiotemporal dopaminergic signaling landscape, while providing a clear set of theoretical predictions and testable hypotheses. We regard our biophysical modeling as the first step towards further experimental investigations required to test our results in animal models and ultimately in PD patients. We hope that the present work will lay the groundwork for new research directions within both basic and clinical neurosciences, aimed at better understanding and treating PD. 


\section{References}

Ba, F., and Martin, W.R.W. (2015). Dopamine transporter imaging as a diagnostic tool for parkinsonism and related disorders in clinical practice. Park. Relat. Disord. 21, 87-94.

Bernheimer, H., Birkmayer, W., Hornykiewicz, O., Jellinger, K., and Seitelberger, F. (1973). Brain dopamine and the syndromes of Parkinson and Huntington Clinical, morphological and neurochemical correlations. J. Neurol. Sci. 20, 415-455.

Bolam, J.P., and Pissadaki, E.K. (2012). Living on the edge with too many mouths to feed: Why dopamine neurons die. Mov. Disord. 27, 1478-1483.

Brotchie, J., and Fitzer-Attas, C. (2009). Mechanisms compensating for dopamine loss in early Parkinson disease. Neurology 72, S32-S38.

Burke, D.A., Rotstein, H.G., and Alvarez, V.A. (2017). Striatal Local Circuitry: A New Framework for Lateral Inhibition. Neuron 96, 267-284.

Chu, Y., and Kordower, J.H. (2015). The Prion Hypothesis of Parkinson's Disease. Curr. Neurol. Neurosci. Rep. 15, 28.

Cui, G., Jun, S.B., Jin, X., Pham, M.D., Vogel, S.S., Lovinger, D.M., and Costa, R.M. (2013). Concurrent activation of striatal direct and indirect pathways during action initiation. Nature 494, 238-242.

Damier, P., Hirsch, E.C., Agid, Y., and Graybiel, A.M. (1999). The substantia nigra of the human brain. II. Patterns of loss of dopamine-containing neurons in Parkinson's disease. Brain 122, Pt 8, 1437-1448.

Doucet, G., Descarries, L., and Garcia, S. (1986). Quantification of the dopamine innervation in adult rat neostriatum. Neuroscience 19, 427-445.

Dreyer, J.K. (2014). Three Mechanisms by which Striatal Denervation Causes Breakdown of Dopamine Signaling. J. Neurosci. 34, 12444-12456.

Dreyer, J.K., Herrik, K.F., Berg, R.W., and Hounsgaard, J.D. (2010). Influence of Phasic and Tonic Dopamine Release on Receptor Activation. J. Neurosci. 30, 14273-14283.

Eckert, T., Tang, C., and Eidelberg, D. (2007). Assessment of the progression of Parkinson's disease: a metabolic network approach. Lancet Neurol. 6, 926-932. 
510

511

512

513

514

515

516

517

518

519

520

521

522

523

524

525

526

527

528

529

530

531

532

533

534

535

536

Fearnley, J.M., and Lees, A.J. (1991). Ageing and Parkinson's disease: substantia nigra regional selectivity. Brain 114, Pt 5, 2283-2301.

Filippi, L., Manni, C., Pierantozzi, M., Brusa, L., Danieli, R., Stanzione, P., and Schillaci, O. (2006). 123I-FP-CIT in progressive supranuclear palsy and in parkinson's disease: a SPECT semiquantitative study. Nucl. Med. Commun. 27, 381-386.

Ford, C.P. (2014). The role of D2-autoreceptors in regulating dopamine neuron activity and transmission. Neuroscience 282, 13-22.

Freeze, B.S., Kravitz, A. V., Hammack, N., Berke, J.D., and Kreitzer, A.C. (2013). Control of Basal Ganglia Output by Direct and Indirect Pathway Projection Neurons. J. Neurosci. 33, 18531-18539.

Gajera, C.R., Fernandez, R., Postupna, N., Montine, K.S., Fox, E.J., Tebaykin, D., Angelo, M., Bendall, S.C., Keene, C.D., and Montine, T.J. (2019). Mass synaptometry: High-dimensional multi parametric assay for single synapses. J. Neurosci. Methods 312, 73-83.

Gajera, C.R., Fernandez, R., Montine, K.S., Fox, E.J., Mrdjen, D., Postupna, N.O., Keene, C.D., Bendall, S.C., and Montine, T.J. (2021). Mass-tag barcoding for multiplexed analysis of human synaptosomes and other anuclear events. Cytom. Part A 99, 939-945.

Grace, A.A., and Bunney, B.S. (1984a). The control of firing pattern in nigral dopamine neurons: single spike firing. J. Neurosci. 4, 2866-2876.

Grace, A.A., and Bunney, B.S. (1984b). The control of firing pattern in nigral dopamine neurons: burst firing. J. Neurosci. 4, 2877-2890.

Greenbaum, L., Lorberboym, M., Melamed, E., Rigbi, A., Barhum, Y., Kohn, Y., Khlebtovsky, A., Lerer, B., and Djaldetti, R. (2013). Perspective: Identification of genetic variants associated with dopaminergic compensatory mechanisms in early Parkinson's disease. Front. Neurosci. 7, 52.

Greenland, J.C., Williams-Gray, C.H., and Barker, R.A. (2018). The clinical heterogeneity of Parkinson's disease and its therapeutic implications. Eur. J. Neurosci.

Guttman, M., and Seeman, P. (1985). L-DOPA reverses the elevated density of D2 dopamine receptors in Parkinson's diseased striatum. J. Neural Transm. 64, 93-103. 
Hardman, C.D., Henderson, J.M., Finkelstein, D.I., Horne, M.K., Paxinos, G., and Halliday, G.M. (2002). Comparison of the basal ganglia in rats, marmosets, macaques, baboons, and humans: volume and neuronal number for the output, internal relay, and striatal modulating nuclei. J. Comp. Neurol. 445, 238-255.

Hauser, R.A. (2009). Levodopa: Past, Present, and Future. Eur. Neurol. 62, 1-8.

Hirtz, D., Thurman, D.J., Gwinn-Hardy, K., Mohamed, M., Chaudhuri, A.R., and Zalutsky, R. (2007). How common are the "common" neurologic disorders? Neurology 68, 326-337.

Hjorth, J., Kozlov, A., Carannante, I., Frost Nylén, J., Lindroos, R., Johansson, Y., Tokarska, A., Dorst, M.C., Suryanarayana, S.M., Silberberg, G., et al. (2020). The microcircuits of striatum in silico. Proc. Natl. Acad. Sci. U.S.A.

Jenner, P. (2003). Oxidative stress in Parkinson's disease. Ann. Neurol. 53, S26-S38.

Kim, Y.J., Ichise, M., Ballinger, J.R., Vines, D., Erami, S.S., Tatschida, T., and Lang, A.E. (2002). Combination of dopamine transporter and D2 receptor SPECT in the diagnostic evaluation of PD, MSA, and PSP. Mov. Disord. 17, 303-312.

Knudsen, G.M., Karlsborg, M., Thomsen, G., Krabbe, K., Regeur, L., Nygaard, T., Videbæk, C., and Werdelin, L. (2004). Imaging of dopamine transporters and D2 receptors in patients with Parkinson's disease and multiple system atrophy. Eur. J. Nucl. Med. Mol. Imaging 31, 1631-1638.

Kravitz, A. V., Freeze, B.S., Parker, P.R.L., Kay, K., Thwin, M.T., Deisseroth, K., and Kreitzer, A.C. (2010). Regulation of parkinsonian motor behaviours by optogenetic control of basal ganglia circuitry. Nature 466, 622-626.

Kreitzer, A.C. (2009). Physiology and Pharmacology of Striatal Neurons. Annu. Rev. Neurosci. 32, 127-147.

Kreitzer, A.C., and Malenka, R.C. (2008). Striatal plasticity and basal ganglia circuit function. Neuron 60, 543554.

Lahiri, A.K., and Bevan, M.D. (2020). Dopaminergic Transmission Rapidly and Persistently Enhances Excitability of D1 Receptor-Expressing Striatal Projection Neurons. Neuron 106, 277-290.

Lee, C.S., Samii, A., Sossi, V., Ruth, T.J., Schulzer, M., Holden, J.E., Wudel, J., Pal, P.K., de la Fuente-Fernandez, R., Calne, D.B., et al. (2000). In vivo positron emission tomographic evidence for compensatory changes in presynaptic dopaminergic nerve terminals in Parkinson's disease. Ann. Neurol. 47, 493-503. 
Lee, T., Seeman, P., Rajput, A., Farley, I.J., and Hornykiewicz, O. (1978). Receptor basis for dopaminergic supersensitivity in Parkinson's disease. Nature 273, 59-61.

Lewis, S.J.G., Foltynie, T., Blackwell, A.D., Robbins, T.W., Owen, A.M., and Barker, R.A. (2005). Heterogeneity of Parkinson's disease in the early clinical stages using a data driven approach. J. Neurol. Neurosurg. Psychiatry 76, 343-348.

LeWitt, P.A. (2008). Levodopa for the Treatment of Parkinson's Disease. N. Engl. J. Med. 359, 2468-2476.

Lippert, R.N., Cremer, A.L., Edwin Thanarajah, S., Korn, C., Jahans-Price, T., Burgeno, L.M., Tittgemeyer, M., Brüning, J.C., Walton, M.E., and Backes, H. (2019). Time-dependent assessment of stimulus-evoked regional dopamine release. Nat. Commun. 10, 336.

Liu, C., Kershberg, L., Wang, J., Schneeberger, S., and Kaeser, P.S. (2018). Dopamine Secretion Is Mediated by Sparse Active Zone-like Release Sites. Cell 172, 706-718.

Liu, Y., Fan, J.H., Gao, X., Ma, L., Qiao, Y.L., and Zhang, L. (2015). The natural progression of Parkinson's disease in a small cohort with 15 drug-naïve patients. Chin. Med. J. (Engl). 128, 1761-1764.

Ma, S.Y., Röyttä, M., Rinne, J.O., Collan, Y., and Rinne, U.K. (1997). Correlation between neuromorphometry in the substantia nigra and clinical features in Parkinson's disease using disector counts. J. Neurol. Sci. 151, 8387.

Matsuda, W., Furuta, T., Nakamura, K.C., Hioki, H., Fujiyama, F., Arai, R., and Kaneko, T. (2009). Single nigrostriatal dopaminergic neurons form widely spread and highly dense axonal arborizations in the neostriatum. J. Neurosci. 29, 444-453.

Mazzoni, P., Hristova, A., and Krakauer, J.W. (2007). Why Don't We Move Faster? Parkinson's Disease, Movement Vigor, and Implicit Motivation. J. Neurosci. 27, 7105-7116.

Michel, P.P., Hirsch, E.C., and Hunot, S. (2016). Understanding Dopaminergic Cell Death Pathways in Parkinson Disease. Neuron 90, 675-691.

Niccolini, F., Foltynie, T., Reis Marques, T., Muhlert, N., Tziortzi, A.C., Searle, G.E., Natesan, S., Kapur, S., Rabiner, E.A., Gunn, R.N., et al. (2015). Loss of phosphodiesterase 10A expression is associated with progression and severity in Parkinson's disease. Brain 138, 3003-3015. 
Nisenbaum, E.S., Xu, Z.C., and Wilson, C.J. (1994). Contribution of a slowly inactivating potassium current to the transition to firing of neostriatal spiny projection neurons. J. Neurophysiol. 71, 1174-1189.

Patriarchi, T., Cho, J.R., Merten, K., Howe, M.W., Marley, A., Xiong, W.-H., Folk, R.W., Broussard, G.J., Liang, R., Jang, M.J., et al. (2018). Ultrafast neuronal imaging of dopamine dynamics with designed genetically encoded sensors. Science 360, eaat4422.

Pereira, D.B., Schmitz, Y., Mészros, J., Merchant, P., Hu, G., Li, S., Henke, A., Lizardi-Ortiz, J.E., Karpowicz, R.J., Morgenstern, T.J., et al. (2016). Fluorescent false neurotransmitter reveals functionally silent dopamine vesicle clusters in the striatum. Nat. Neurosci. 19, 578-586.

Poulin, J.-F., Caronia, G., Hofer, C., Cui, Q., Helm, B., Ramakrishnan, C., Chan, C.S., Dombeck, D.A., Deisseroth, K., and Awatramani, R. (2018). Mapping projections of molecularly defined dopamine neuron subtypes using intersectional genetic approaches. Nat. Neurosci. 21, 1260-1271.

Prusiner, S.B. (2012). A Unifying Role for Prions in Neurodegenerative Diseases. Science (80-. ). 336, 1511-1513.

Rasmussen, R., Jensen, M.H., and Heltberg, M.L. (2017). Chaotic Dynamics Mediate Brain State Transitions, Driven by Changes in Extracellular Ion Concentrations. Cell Syst. 5, 591-603.

Richfield, E.K., Penney, J.B., and Young, A.B. (1989). Anatomical and affinity state comparisons between dopamine D1 and D2 receptors in the rat central nervous system. Neuroscience 30, 767-777.

Rodriguez-Oroz, M.C., Jahanshahi, M., Krack, P., Litvan, I., Macias, R., Bezard, E., and Obeso, J.A. (2009). Initial clinical manifestations of Parkinson's disease: features and pathophysiological mechanisms. Lancet Neurol. $8,1128-1139$.

Salat, D., and Tolosa, E. (2013). Levodopa in the treatment of Parkinson's disease: Current status and new developments. J. Parkinsons. Dis. 3, 255-269.

Shen, W., Hernandez-Lopez, S., Tkatch, T., Held, J.E., and Surmeier, D.J. (2004). Kv1.2-Containing K+ Channels Regulate Subthreshold Excitability of Striatal Medium Spiny Neurons. J. Neurophysiol. 91, 1337-1349.

Sippy, T., Lapray, D., Crochet, S., and Petersen, C.C.H. (2015). Cell-Type-Specific Sensorimotor Processing in Striatal Projection Neurons during Goal-Directed Behavior. Neuron 88, 298-305.

Sulzer, D. (2007). Multiple hit hypotheses for dopamine neuron loss in Parkinson's disease. Trends Neurosci. 30, 244-250. 
Surmeier, D.J., and Kitai, S.T. (1993). D1 and D2 dopamine receptor modulation of sodium and potassium currents in rat neostriatal neurons. Prog. Brain Res. 99, 309-324.

Surmeier, D.J., Bargas, J., Hemmings, H.C., Nairn, A.C., and Greengard, P. (1995). Modulation of calcium currents by a D1 dopaminergic protein kinase/phosphatase cascade in rat neostriatal neurons. Neuron 14, 385-397.

Surmeier, J.D., Ding, J., Day, M., Wang, Z., and Shen, W. (2007). D1 and D2 dopamine-receptor modulation of striatal glutamatergic signaling in striatal medium spiny neurons. Trends Neurosci. 30, 228-235.

Surmeier, J.D., Obeso, J.A., and Halliday, G.M. (2017). Selective neuronal vulnerability in Parkinson disease. Nat. Rev. Neurosci. 18, 101-113.

Tatsuki, F., Sunagawa, G.A., Shi, S., Susaki, E.A., Yukinaga, H., Perrin, D., Sumiyama, K., Ukai-Tadenuma, M., Fujishima, H., Ohno, R., et al. (2016). Involvement of Ca2+-Dependent Hyperpolarization in Sleep Duration in Mammals. Neuron 90, 70-85.

Varrone, A., Marek, K.L., Jennings, D., Innis, R.B., and Seibyl, J.P. (2001). [123I]ß-CIT SPECT imaging demonstrates reduced density of striatal dopamine transporters in Parkinson's disease and multiple system atrophy. Mov. Disord. 16, 1023-1032.

Wang, L., Zhang, Q., Li, H., and Zhang, H. (2012). SPECT molecular imaging in Parkinson's disease. J. Biomed. Biotechnol. 2012, 412486.

Wickens, J.R., and Wilson, C.J. (1998). Regulation of action-potential firing in spiny neurons of the rat neostriatum in vivo. J. Neurophysiol. 79, 2358-2364.

Ziebell, M., Andersen, B.B., Thomsen, G., Pinborg, L.H., Karlsborg, M., Hasselbalch, S.G., and Knudsen, G.M. (2012). Predictive value of dopamine transporter SPECT imaging with [123I]PE2I in patients with subtle parkinsonian symptoms. Eur. J. Nucl. Med. Mol. Imaging 39, 242-250.

Zigmond, M.J. (1997). Do Compensatory Processes Underlie the Preclinical Phase of Neurodegenerative Disease? Insights from an Animal Model of Parkinsonism. Neurobiol. Dis. 4, 247-253.

Zigmond, M.J., Abercrombie, E.D., Berger, T.W., Grace, A.A., and Stricker, E.M. (1990). Compensations after lesions of central dopaminergic neurons: some clinical and basic implications. Trends Neurosci. 13, 290296. 
644 Fig. 1. Functional and spatial characterization of DA signaling in the healthy human striatum. A, Diagram

645 of dopaminergic innervation and signaling in the human striatum. B, Diagram of dopaminergic regulation of D1646 and D2-SPNs, parts of the direct and indirect pathway, respectively. C, Trace showing DA signaling and the 647 underlying dopaminergic neuronal firing pattern. D, Illustration of overlapping dopaminergic axonal arbors 648 belonging to the same CAC. E, Visualization of dopaminergic axonal arbors in the striatum; each arbor center is 649 marked with a circle. For visibility, only $10 \%$ of arbors are shown. Red sphere shows the area subsumed by an 650 arbor from one neuron. Notice that all arbors belong to the same CAC, represented by them all having the same 651 color. F, Heatmap of the distribution of overlapping arbors in the two-dimensional plane denoted in E. G, 652 Distribution of the number of overlapping arbors for each individual arbor. H, Distribution of the smallest 653 distance to the nearest neighboring arbor center for each arbor. Inset: smallest distance to nearest neighboring 654 arbor center for the most isolated arbors found using Voronoi tessellation. DA, dopamine; SNc, substantia nigra 655 pars compacta; D1, D1-class dopamine receptor; D2, D2-class dopamine receptor; SPN, spiny projection neuron; 656 CAC, contiguous arbor classes. See also Extended Data Equations Fig. 1-1. 
659 Fig. 2. Different denervation patterns break down the dopaminergic network with distinct evolutions.

660 A-C, Diagrams of network mechanism for RD, PLD, and SID. The color of each dopaminergic neuron (circle) 661 corresponds to probability of death. In C, dotted lines denote overlap of arbors. D, Visualization of the 662 dopaminergic axonal arbor network following RD, PLD and SID. Colors correspond to separate CACs. E, 663 Distributions of the number of overlapping arbors for each individual arbor. f, Distributions of the number of 664 arbors in each CAC. G, Distributions of the smallest distance to the nearest neighboring arbor center for each 665 arbor. Dotted line denotes threshold for classifying isolated areas. In E-G, denervation is $80 \%$. H, Fraction of 666 remaining arbors as a function of time. I, Fraction of arbors belonging to the largest CAC as a function of 667 denervation. J, Fraction of striatal space with smallest distance to nearest arbor larger than $0.1 \mathrm{~mm}$ (isolated 668 area) as a function of denervation. In $\mathbf{H}-\mathbf{J}$, full line is mean, and shading is standard deviation. RD, random denervation; PLD, prion-like denervation; SID, stress-induced denervation; CAC, contiguous arbor classes; AU, arbitrary unit. See also Extended Data Fig. 2-1.

671 
673 Fig. 3. Dopaminergic denervation affects cAMP signaling and excitability of striatal SPNs. A, Diagram of 674 how DA stimulates and inhibits the production of cAMP in D1- and D2-SPNs, respectively. B, Traces showing 675 cAMP in D1- and D2-SPNs as a function of DA signaling. C, Maximal cAMP concentration in D1- and D2-SPNs 676 during dopaminergic phasic firing and firing pauses, respectively, as a function of the number of dopaminergic 677 terminals. D-K, Membrane potential dynamics of D1- and D2- SPNs in response to synaptic barrages (D, H), 678 cAMP stimulation (E, I), synaptic barrages in combination with cAMP stimulation in the healthy state (F, J), or 679 synaptic barrages in combination with cAMP stimulation in the 75\% denervated state (G, K). Raster plots show 680 the firing rate across time for 100 simulated neurons in each condition. DA, dopamine; D1, D1-class dopamine 681 receptor; D2, D2-class dopamine receptor; SPN, spiny projection neuron; SNc, substantia nigra pars compacta; 682 cAMP, cyclic adenosine monophosphate. See also Extended Data Fig. 3-1. 
685 Fig. 4. Distinct denervation patterns differentially affect global striatal SPN firing activity. A-B, Maximal 686 firing activity of D1- and D2-SPNs across space in the healthy and 75\% denervated striatum for the three 687 denervation patterns. C-D, Spatial mean and standard deviation of maximal firing activity in D1- and D2-SPNs as 688 a function of denervation. RD, random denervation; PLD, prion-like denervation; SID, stress-induced 689 denervation; D1, D1-class dopamine receptor; D2, D2-class dopamine receptor; SPN, spiny projection neuron. 
691 Fig. 5. A dual presynaptic compensation strategy preserves DA signaling in the denervated striatum. A,

692 Diagrams of mechanisms of the ERC, DUC, and DEC models. B, Traces showing cAMP in D1- and D2-SPNs as a 693 function of DA signaling at $80 \%$ denervation in the compensation models. C-D, Spatial mean and standard 694 deviation of maximal firing activity in D1- and D2-SPNs as a function of denervation pattern and compensation 695 model. E, Spatial mean of maximal firing activity in D1- and D2-SPNs as a function of denervation and 696 compensation model in the randomly denervated striatum. ERC, enhanced release compensation; DUC, 697 decreased uptake compensation; DEC, dual enhanced compensation; NC, no compensation; DA, dopamine; 698 cAMP, cyclic adenosine monophosphate; D1, D1-class dopamine receptor; D2, D2-class dopamine receptor; SPN, 699 spiny projection neuron; RD, random denervation; PLD, prion-like denervation; SID, stress-induced denervation. 700 See also Extended Data Figs. 5-1 and 5-2.

701 
702 Extended Data Equations. Mathematical models and algorithms. Folder containing a detailed description of 703 all mathematical derivations and formulations, biophysical models, and algorithms employed for the study. 704 
705 Extended Data Fig 1-1. Model parameters. Table summarizing the biophysical parameter values used for the 706 respective computational models. 
707 Extended Data Fig 2-1. Spatial denervation evolutions are robust to changes in key parameters. A, Effects

708 of varying the volume of axonal arbors (radius: $0.45 \mathrm{~mm}, 0.5 \mathrm{~mm}$, or $0.55 \mathrm{~mm}$ ) uniformly across the arbor

709 population in the three denervation models. Upper: Fraction of arbors belonging to the largest CAC as a function 710 of denervation; lower: fraction of striatal space with smallest distance to nearest arbor larger than $0.1 \mathrm{~mm}$

711 (isolated area) as a function of denervation. B, Same as in A, but with the volume of arbors following a $\delta$ function

$712\left(V_{n}=\delta\left(V_{n}-V_{0}\right)\right)$ versus heterogeneous distribution so the volume follows a normal distribution $\left(V_{n}=\frac{V_{0}}{10} \times\right.$ $713 N(0,1))$. For both cases $V_{0}=\frac{4}{3} \pi r_{0}$ where $r_{0}$ is the standard radius $\left(r_{0}=0.5 \mathrm{~mm}\right)$. C, Same as in A, but for 714 different numbers of dopaminergic neurons in the healthy state (80 000, 100000 or 130000 neurons). In A-C, 715 full line is the mean and shading is the standard deviation. RD, random denervation; PLD, prion-like 716 denervation; SID, stress-induced denervation; CAC, contiguous arbor class. 
717 Extended Data Fig. 3-1. Distinct denervation patterns differentially affect local and global striatal SPN

718 firing activity in the Izhikevich model. A, Membrane potential of D1-SPN (left) and D2-SPN (right) in the 719 healthy and 75\% denervated striatum in response to DA signaling, modelled using the Izhikevich model. B-C, 720 Maximal firing activity of D1- and D2-SPNs across space in the healthy and 75\% denervated striatum for the 721 three denervation patterns: RD, PLD, and SID. D-E, Spatial mean and standard deviation of maximum firing 722 activity in D1- and D2-SPNs as a function of denervation. SPN, spiny projection neuron; D1, D1-class dopamine 723 receptor; D2, D2-class dopamine receptor; DA, dopamine.

724

725 
726 Extended Data Fig. 5-1. Three distinct postsynaptic mechanisms fail to preserve DA signaling in the

727 denervated striatum. A-C, Diagrams of the modelled postsynaptic compensatory mechanisms: increased D2

728 expression (A), enhanced D1 and D2 sensitivity (B), and suppressed cAMP degradation in D1- and D2-SPNs

729 mediated by, for example, a PDE inhibitor (PDE-I) (C). D-F, Example traces showing cAMP in D1- and D2-SPNs

730 as a function of DA signaling at $0 \%$ and $80 \%$ denervation in the different postsynaptic compensation models.

731 DA, dopamine; D2, D2-class dopamine receptor; D1, D1-class dopamine receptor; cAMP, cyclic adenosine 732 monophosphate; SPN, spiny projection neuron; PDE, phosphodiesterase.

733 
734 Extended Data Fig. 5-2. A dual presynaptic compensation strategy preserves SPN firing activity in the 735 Izhikevich model in spite of severe denervation. A-B, Spatial mean and standard deviation of maximum 736 firing activity in D1- and D2-SPNs as a function of denervation pattern (RD, PLD, and SID), and compensation 737 model (ERC, DUC, and DEC). C, Spatial mean of maximum firing activity in D1- and D2-SPNs as a function of 738 denervation and compensation model in the randomly denervated striatum. SPN, spiny projection neuron; D1, 739 D1-class dopamine receptor; D2, D2-class dopamine receptor; RD, random denervation; PLD, prion-like 740 denervation; SID, stress-induced denervation; ERC, enhanced release compensation; DUC, decreased uptake 741 compensation; DEC, dual enhanced compensation. 
A

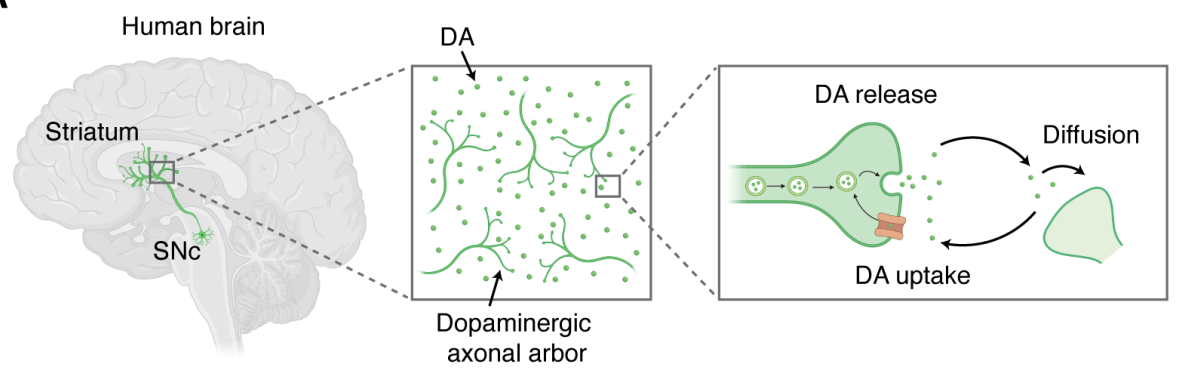

B

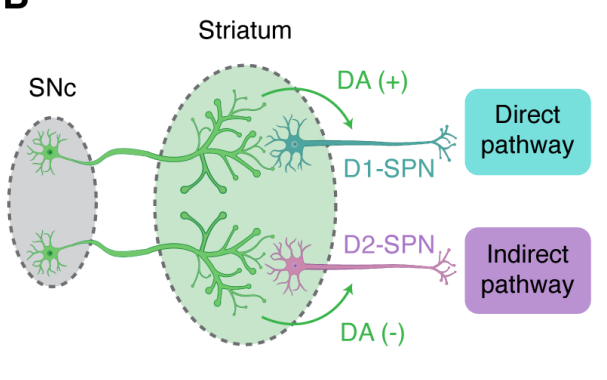

C

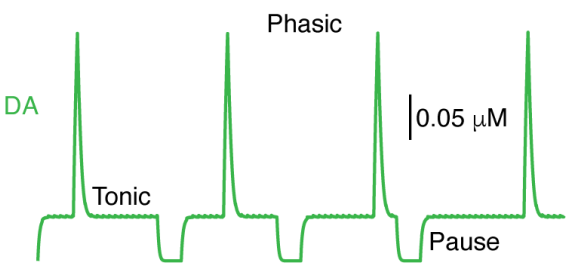

(

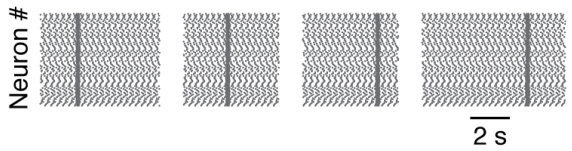

E

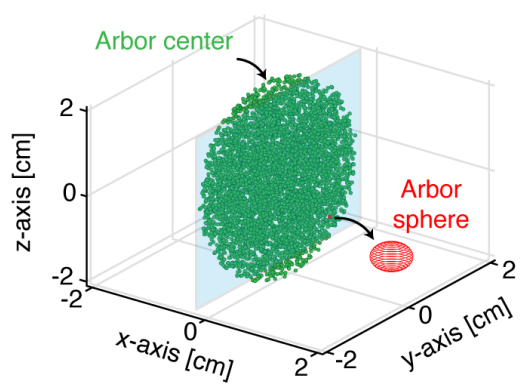

D

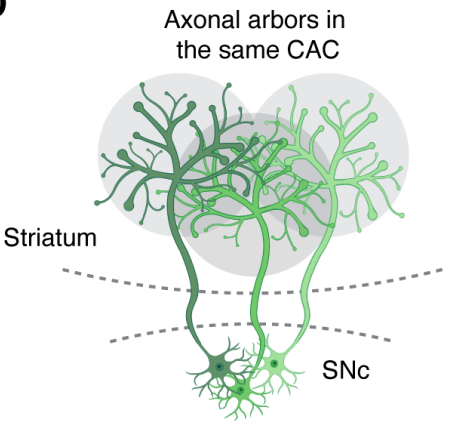

F

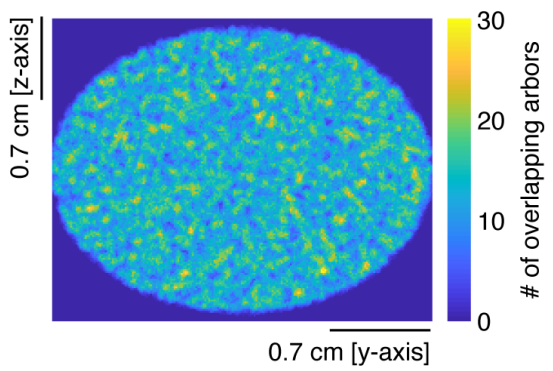

G

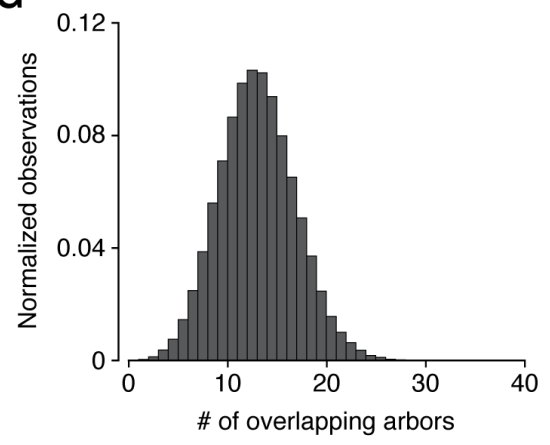

H

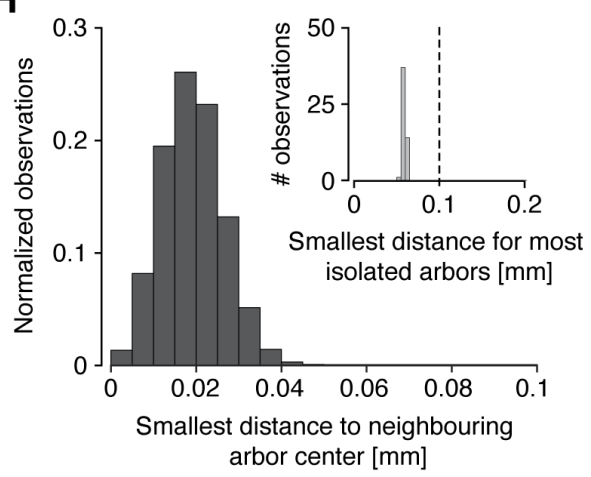



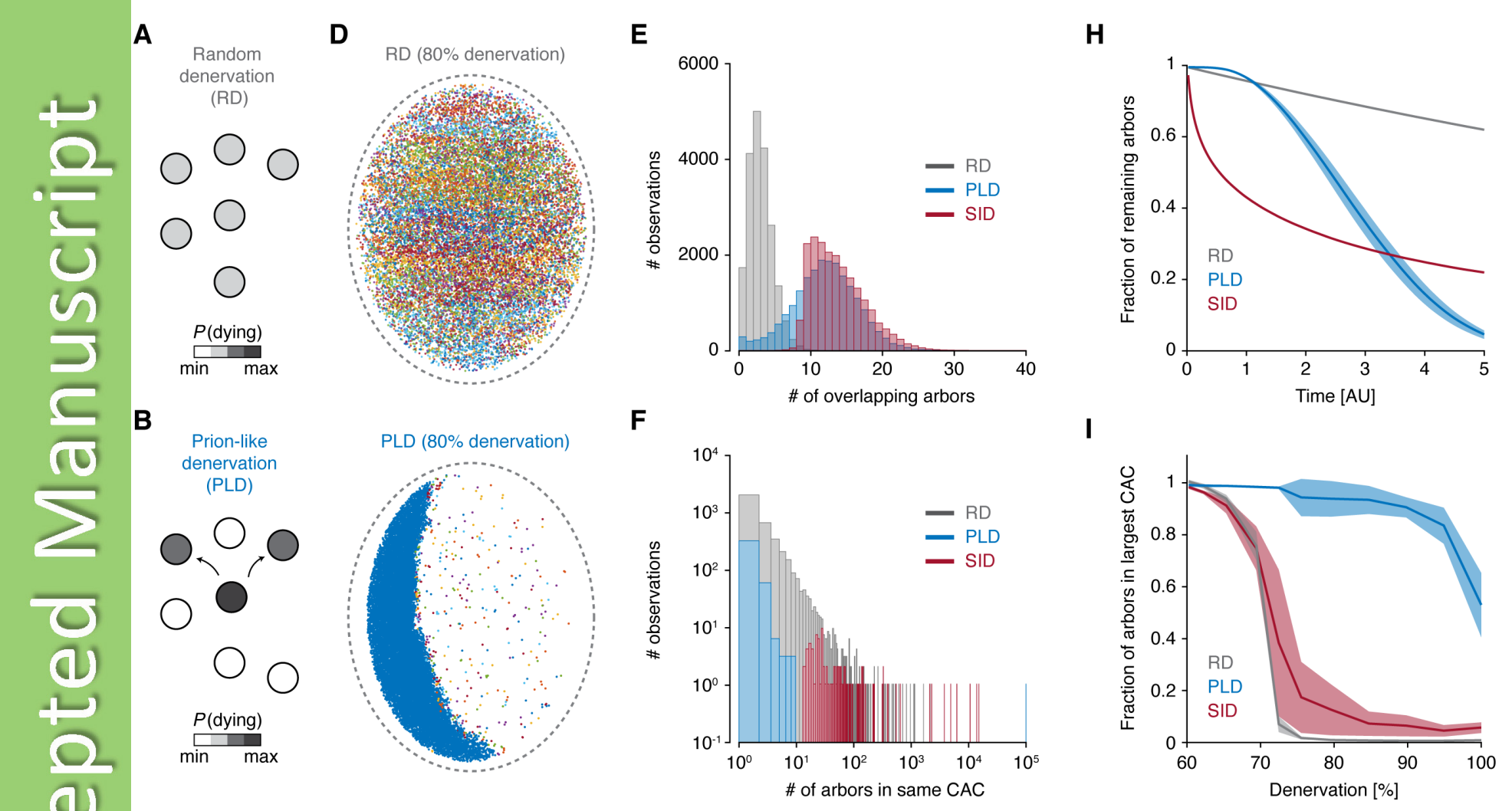

Prion-like
denervatio

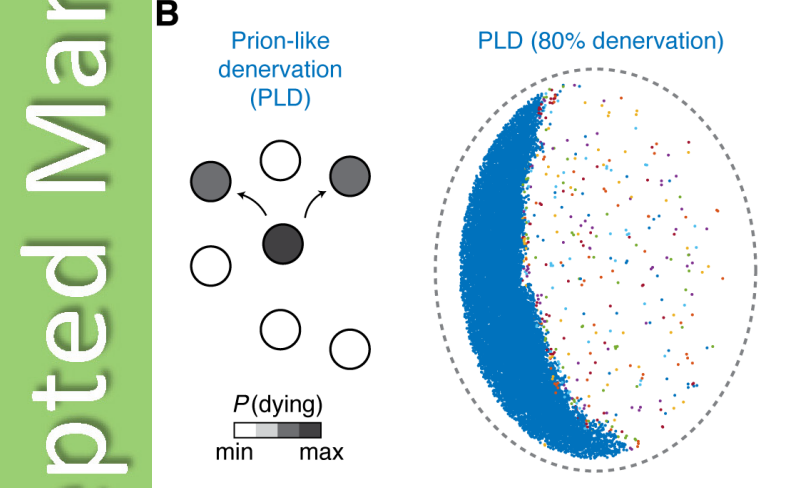

(U) C Stress-induced
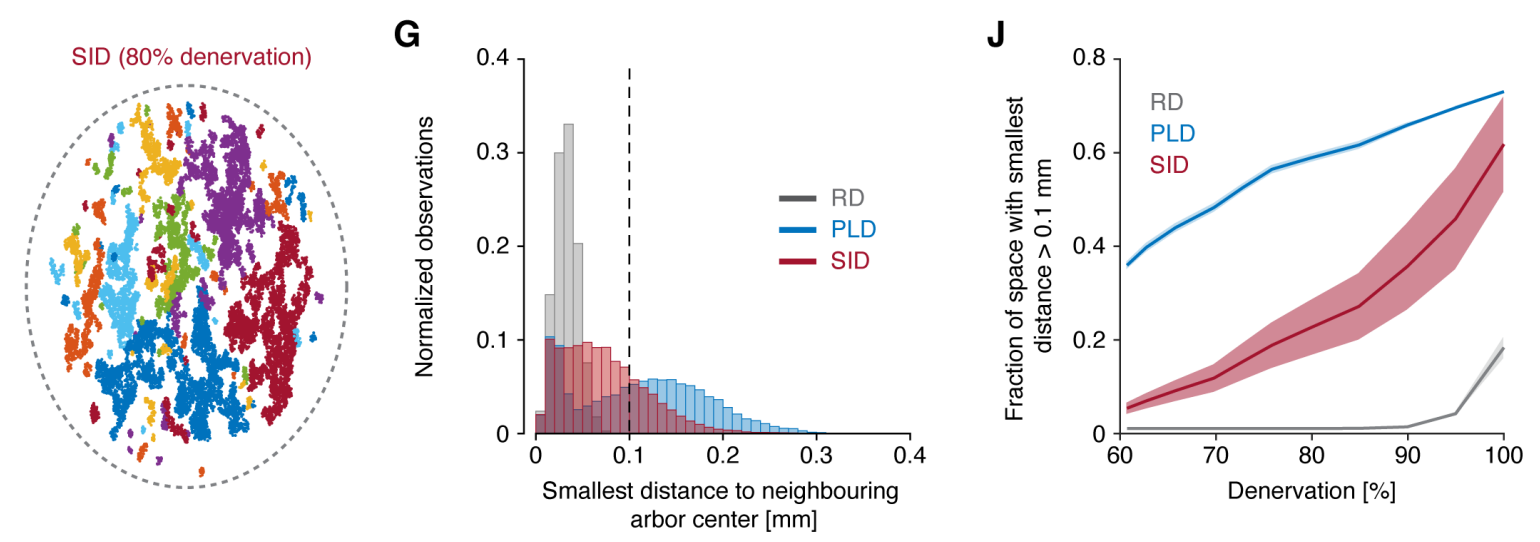


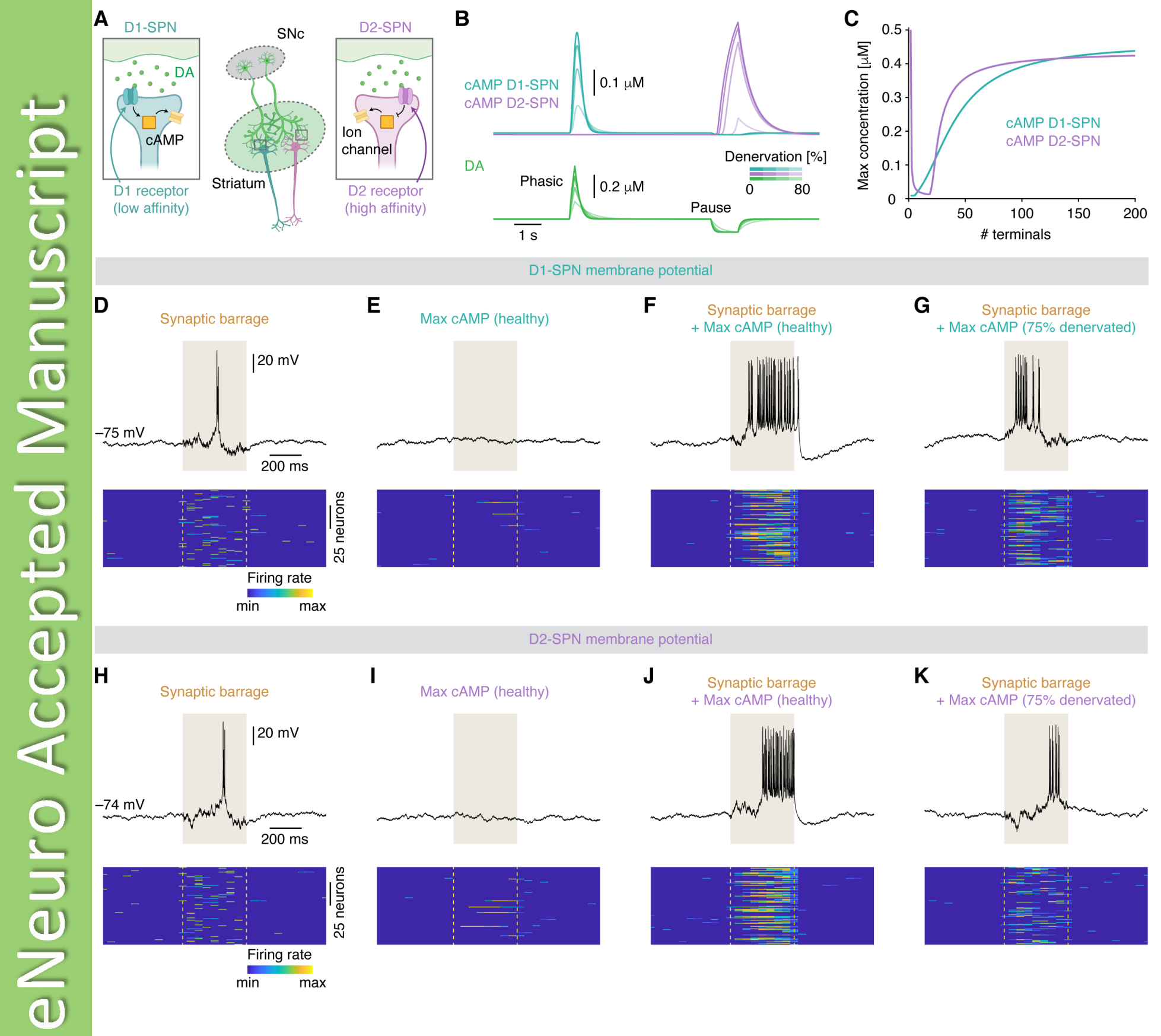


A

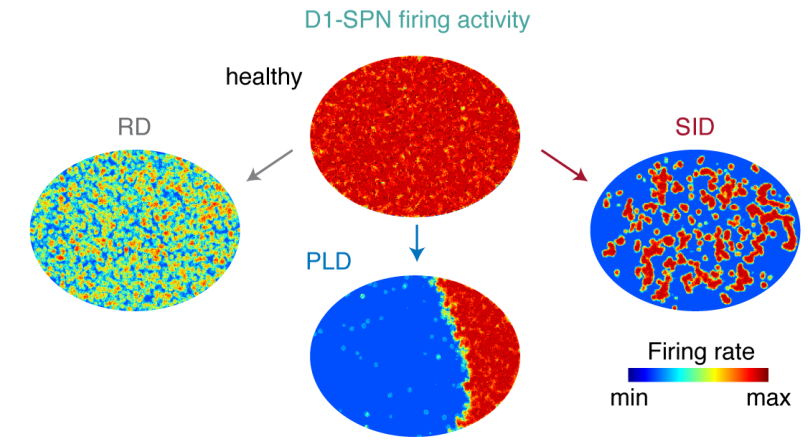

B

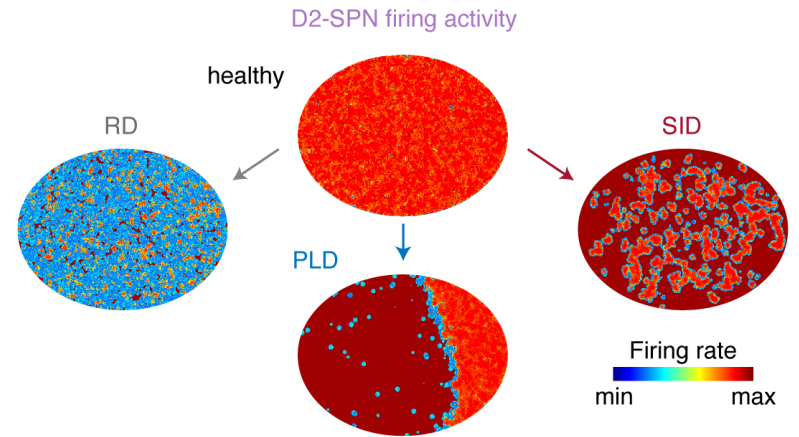

D
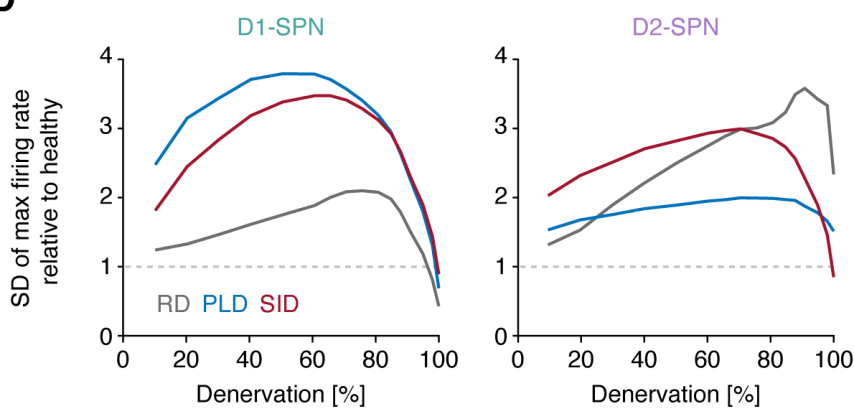

\section{( ) \\ 난 \\ (d)


A

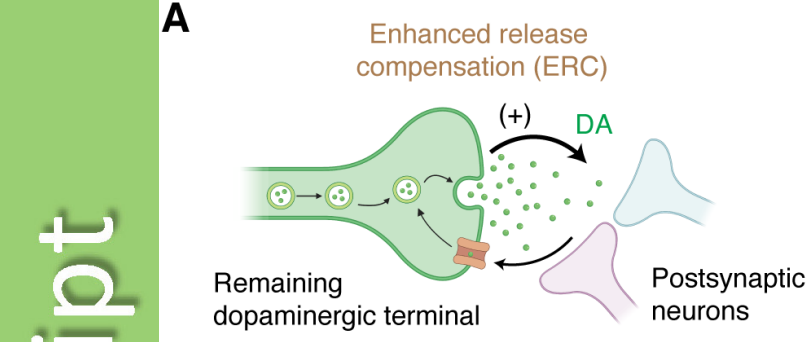

B

$80 \%$ denervation with ERC

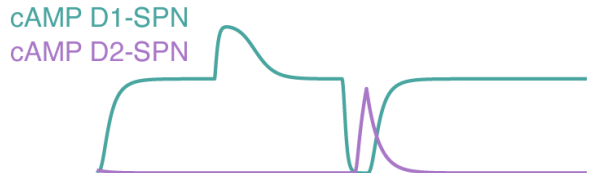

DA

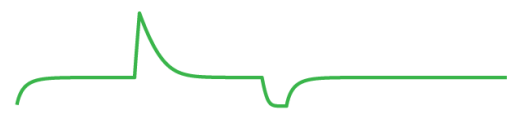

C

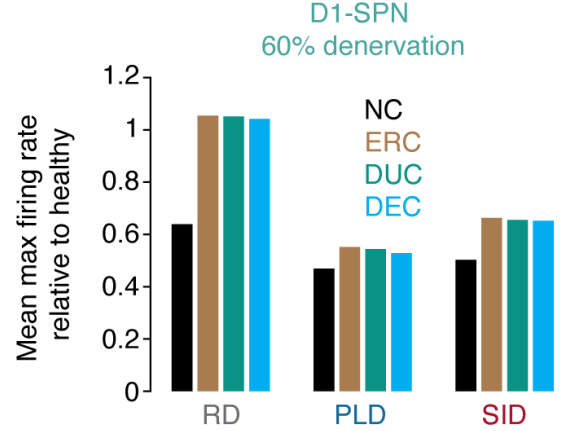

D

(2)

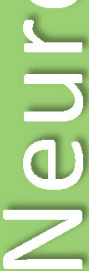

(U)

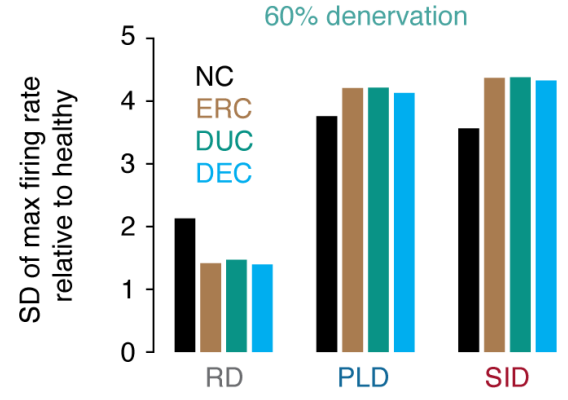

D1-SPN

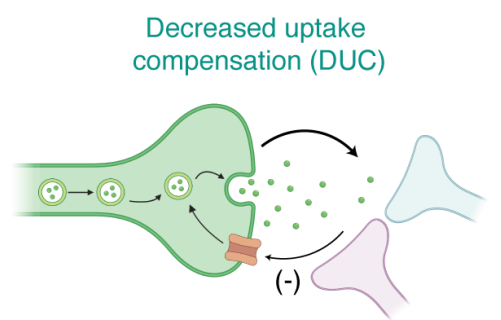

$80 \%$ denervation with DUC
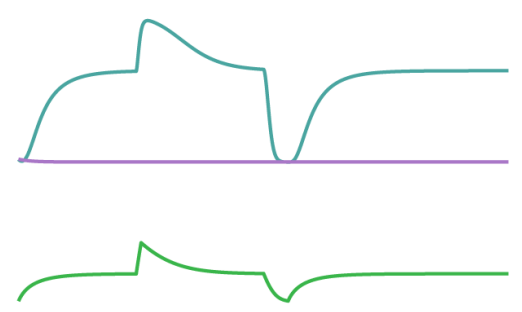

E

D2-SPN

$60 \%$ denervation

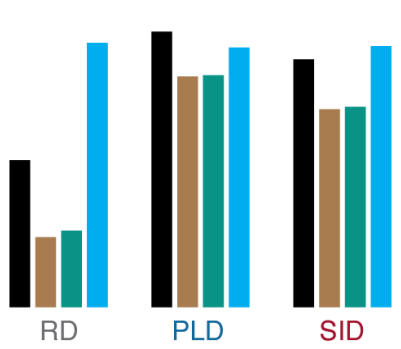

D2-SPN

$60 \%$ denervation

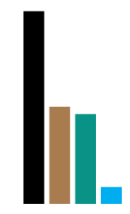

$\mathrm{RD}$

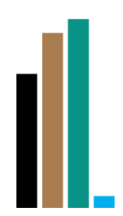

PLD

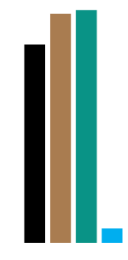

SID
Dual enhanced compensation (DEC)

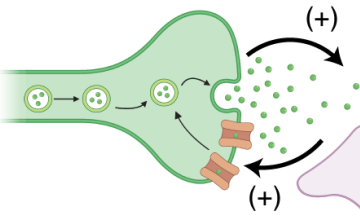

$80 \%$ denervation with DEC
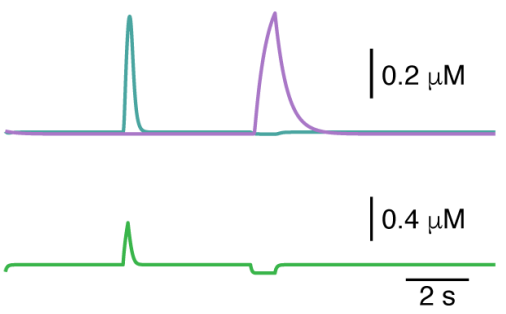

D1-SPN

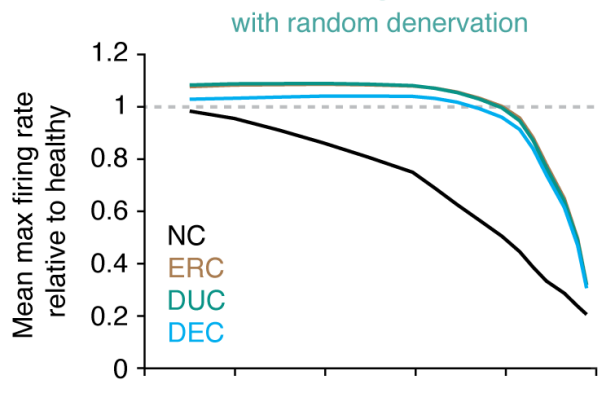

D2-SPN

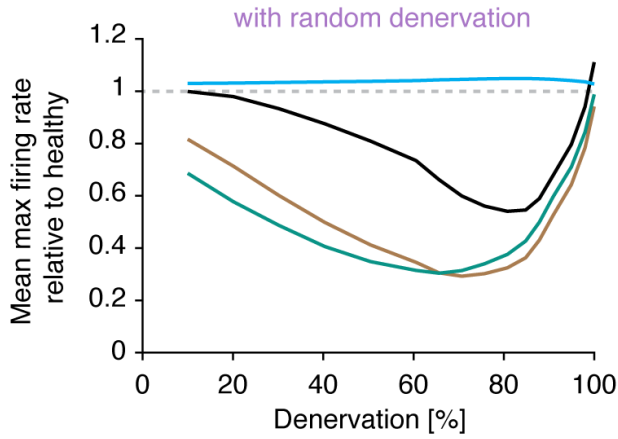

